\title{
Interventions for improving pregnancy outcomes in antenatally diagnosed or suspected morbidly adherent placenta
}

\author{
Rinku Sengupta \\ Alexis C. Gimovsky \\ Thomas Jefferson University \\ Shuqin Wei \\ Université de Montréal \\ Anthony Akinloye Bamigboye \\ University of the Witwatersrand \\ Vincenzo Berghella \\ Part of the Obstetrics and Gynecology Commons \\ Let us know how access to this document benefits you
}

\section{Recommended Citation}

Sengupta, Rinku; Gimovsky, Alexis C.; Wei, Shuqin; Bamigboye, Anthony Akinloye; Berghella, Vincenzo; Amorim Adegboye, Amanda R.; and Fraser, William Donald, "Interventions for improving pregnancy outcomes in antenatally diagnosed or suspected morbidly adherent placenta" (2016). Department of Obstetrics and Gynecology Faculty Papers. Paper 34. https://jdc.jefferson.edu/obgynfp/34

This Article is brought to you for free and open access by the Jefferson Digital Commons. The Jefferson Digital Commons is a service of Thomas Jefferson University's Center for Teaching and Learning (CTL). The Commons is a showcase for Jefferson books and journals, peer-reviewed scholarly publications, unique historical collections from the University archives, and teaching tools. The Jefferson Digital Commons allows researchers and interested readers anywhere in the world to learn about and keep up to date with Jefferson scholarship. This article has been accepted for inclusion in Department of Obstetrics and Gynecology Faculty Papers by an authorized administrator of the Jefferson Digital Commons. For more information, please contact: JeffersonDigitalCommons@jefferson.edu. 


\section{Authors}

Rinku Sengupta, Alexis C. Gimovsky, Shuqin Wei, Anthony Akinloye Bamigboye, Vincenzo Berghella, Amanda R. Amorim Adegboye, and William Donald Fraser 


\section{(E) Cochrane Library}

Cochrane Database of Systematic Reviews

\section{Interventions for improving pregnancy outcomes in antenatally diagnosed or suspected morbidly adherent placenta (Protocol)}

Sengupta R, Gimovsky A, Wei S, Bamigboye AA, Berghella V, Amorim Adegboye AR, Fraser WD

Sengupta R, Gimovsky A, Wei S, Bamigboye AA, Berghella V, Amorim Adegboye AR, Fraser WD.

Interventions for improving pregnancy outcomes in antenatally diagnosed or suspected morbidly adherent placenta.

Cochrane Database of Systematic Reviews 2016, Issue 4. Art. No.: CD012159.

DOI: 10.1002/14651858.CD012159.

www.cochranelibrary.com 
TABLE OF CONTENTS

HEADER . . . . . . . . . . . . . . . . . . . . . . . . . . . . . . . . . . . . 1

ABSTRACT . . . . . . . . . . . . . . . . . . . . . . . . . . . . . . . . . . . . . . . . . .

BACKGROUND . . . . . . . . . . . . . . . . . . . . . . . . . . . . . . . . . . . .

OBJECTIVES . . . . . . . . . . . . . . . . . . . . . . . . . . . . . . . . . . . . . . . . . .

METHODS . . . . . . . . . . . . . . . . . . . . . . . . . . . . . . . . . . . . . . 44

ACKNOWLEDGEMENTS . . . . . . . . . . . . . . . . . . . . . . . . . . . . . . . . . . . . . . . . .

REFERENCES . . . . . . . . . . . . . . . . . . . . . . . . . . . . . . . . . . . . . 9

ADDITIONAL TABLES . . . . . . . . . . . . . . . . . . . . . . . . . . . . . . . . . . . 11

APPENDICES . . . . . . . . . . . . . . . . . . . . . . . . . . . . . . . . . . . . . . . . . .

CONTRIBUTIONS OF AUTHORS . . . . . . . . . . . . . . . . . . . . . . . . . . . . . . . . . . . . 13

DECLARATIONS OF INTEREST . . . . . . . . . . . . . . . . . . . . . . . . . . . . . . . . . . . . . . .

Interventions for improving pregnancy outcomes in antenatally diagnosed or suspected morbidly adherent placenta (Protocol)

Copyright $\odot 2016$ The Cochrane Collaboration. Published by John Wiley \& Sons, Ltd. 


\title{
Interventions for improving pregnancy outcomes in antenatally diagnosed or suspected morbidly adherent placenta
}

\author{
Rinku Sengupta ${ }^{1}$, Alexis Gimovsky ${ }^{2}$, Shuqin $\mathrm{Wei}^{3}$, Anthony A Bamigboye ${ }^{4}$, Vincenzo Berghella ${ }^{5}$, Amanda R Amorim Adegboye ${ }^{6}$, \\ William D Fraser ${ }^{7}$ \\ ${ }^{1}$ Shirley Solihull, UK. ${ }^{2}$ Division of Maternal-Fetal Medicine, Department of Obstetrics and Gynecology, Jefferson Medical College \\ of Thomas Jefferson University, Philadelphia, Pennsylvania, USA. ${ }^{3}$ Département d'Obstétrique-Gynécologie, Université de Montréal, \\ Montréal, Canada. ${ }^{4}$ Department of Obstetrics and Gynecology, School of Clinical Medicine, University of the Witwatersrand, South \\ Africa and Delta State University, Nigeria, Faculty of Health Sciences, Johannesburg, South Africa. ${ }^{5}$ Division of Maternal Fetal Medicine, \\ Department of Obstetrics and Gynecology, Thomas Jefferson University, Philadelphia, Pennsylvania, USA. ${ }^{6}$ University of Westminster, \\ London, UK. ${ }^{7}$ Département d'Obstétrique-Gynécologie, Université de Sherbrooke, Sherbrooke, Canada
}

Contact address: Rinku Sengupta, C/o Cochrane Pregnancy and Childbirth Group, Department of Women's and Children's Health, University of Liverpool, First Floor, Liverpool Women's NHS Foundation Trust, Crown Street, Liverpool, L8 7SS, UK. rinkusengupta@ymail.com.

Editorial group: Cochrane Pregnancy and Childbirth Group.

Publication status and date: New, published in Issue 4, 2016.

Citation: Sengupta R, Gimovsky A, Wei S, Bamigboye AA, Berghella V, Amorim Adegboye AR, Fraser WD. Interventions for improving pregnancy outcomes in antenatally diagnosed or suspected morbidly adherent placenta. Cochrane Database of Systematic Reviews 2016, Issue 4. Art. No.: CD012159. DOI: 10.1002/14651858.CD012159.

Copyright (C) 2016 The Cochrane Collaboration. Published by John Wiley \& Sons, Ltd.

\begin{abstract}
A B S T R A C T
This is the protocol for a review and there is no abstract. The objectives are as follows:

To assess the benefits and harms associated with clinical interventions for improving outcomes in women with antenatally diagnosed or suspected MAP. We will compare conventional caesarean hysterectomy with uterine conservation treatments (together with adjunct measures) in women who have been diagnosed antenatally with, or suspected to have, MAP.
\end{abstract}

\section{B A C K G R O U N D}

Morbidly adherent placenta (MAP) is a serious disorder in pregnancy, causing maternal deaths rates as high as $7 \%$ (Wortman 2013). The incidence of this condition has increased in recent years, possibly due to a global rise in caesarean section rates (Al-Khan 2014; Comstock 2014; Vogel 2015). Caesarean delivery rates of $50 \%$ and above are common in some areas of China and Brazil (Cavallaro 2013; Vogel 2015).
Recently, the World Health Organization (WHO), conducted a worldwide systematic review. They concluded that a rise in caesarean section rates up to $10 \%$ to $15 \%$, was associated with reduced maternal and perinatal mortality. In their subsequent worldwide ecological study, they suggested, this could be due to local factors. As caesarean section rates increased above $10 \%$ and up to $30 \%$, they observed no adverse outcomes on mortality rates. They were unable to comment on rates above $30 \%$. Furthermore, in geographical areas where facilities for caesarean section were not so 
well-developed or safe, the risks were higher (WHO 2015).

\section{Description of the condition}

MAP is a condition where the placental villi (finger like projections from the afterbirth) perforate the inner lining of the uterus (womb). There are three subsets (RCOG 2011):

1. accreta: $(75 \%)$ the placental villi attach to the myometrium (womb muscle);

2. increta: $(18 \%)$ the placental villi invade into the myometrium (womb muscle); and

3. percreta: $(7 \%)$ the placental villi invade through the myometrium (womb muscle) (Berkley 2013; Wortman 2013). Placenta accreta is a term that covers all the above (Perez-Delboy 2014). Some authors have described this condition as 'placental attachment disorders' or PAD (Comstock 2014).

MAP is further subdivided according to the number of placental lobules attached to the uterus:

1. total: all the lobules;

2. partial: two lobules; and

3. focal: one lobule (Wortman 2013).

The earliest description of MAP was in the 1500 s by Plater ( Wortman 2013). This condition was not often seen before the 1970s, as caesarean sections were rare before that time (Berkley 2013; Wortman 2013).

Currently, the incidence is variable and difficult to estimate. It is quoted as one in 90 in Israel (Upson 2014), one in 540 in Thailand (Herath 2012), one in 533 in the United States of America (USA) (Eller 2011; Perez-Delboy 2014), and 1.7 in 10,000 in the United Kingdom (UK) (Fitzpatrick 2014). Importantly, the incidence worldwide has increased to almost 10 times over the last 30 years (Eller 2011; Perez-Delboy 2014).

Identifying women with risk factors is important for the early diagnosis and management of the condition (Bowman 2014). The major risk factors for MAP are: low-lying placenta (praevia) and a history of past womb surgery (e.g. caesarean sections, uterine curettage and myomectomy) (Bowman 2014; Comstock 2014). However, other risk factors, such as age 35 years or older, invitro fertilisation (IVF) pregnancy, high parity, smoking, history of fibroids and abnormalities in the shape of the uterus are important (Berkley 2013; Bowman 2014; Hayes 2011; Perez-Delboy 2014). Prenatal diagnosis of MAP is useful in preventing adverse outcomes for the mother and her baby (Comstock 2014; PalaciosJaraquemada 2013; Weiniger 2013). Diagnosis is performed by ultrasound, which is very sensitive, but not very accurate. Special investigations such as Doppler and magnetic resonance imaging (MRI) are helpful, especially when the placenta is attached to the back wall of the uterus (Garmi 2012; Jauniaux 2012; Weiniger 2013). Nevertheless, there are many cases diagnosed after delivery of the baby. These cases can have serious consequences due to a delay in diagnosis and management. Additionally, inaccurate diagnosis may cause iatrogenic (caused by therapy or intervention) pre- mature births, unwanted extensive surgery and protracted hospital stay (Weiniger 2013). Clinicians must have a high index of suspicion when these cases present as otherwise errors in management, can be fatal to the woman (Berkley 2013; Palacios-Jaraquemada 2013).

Consequences of MAP include: massive haemorrhage, damage to internal body organs, including the bladder, bowel and ureter, blood clotting disorders, blood transfusion-related complications, postoperative blood clots, infections, poor functioning of several body organ systems and death of the mother (Berkley 2013; Palacios-Jaraquemada 2013).

\section{Description of the interventions and how these may work}

There are several interventions for MAP. The main focus of this review will be on caesarean hysterectomy versus uterine preservation. We have described below, each important intervention and how each of these might work.

\section{Description of interventions}

\section{Caesarean hysterectomy}

Surgical management consists of performing a planned hysterectomy after delivering the baby, without attempting removal of the placenta. This is the standard treatment worldwide, including the USA (ACOG 2012; Amsalem 2011; Bowman 2014; Eller 2011; Tan 2013; Wortman 2013). Caesarean hysterectomy, is the preferred treatment for massive obstetric haemorrhage unresponsive to conservative measures. It often involves difficult and prolonged surgery, severe bleeding and complications (Eller 2011). There are several techniques and steps involved in performing this surgery, including the type and place of the uterine incision, leaving the placenta in-situ, removing the placenta completely and other adjunct measures as below.

\section{Caesarean delivery and leaving the placenta in-situ}

This intervention involves caesarean section for delivery of the baby, followed by conservative management of the placenta, which is left in place, without any attempt at removal. This intervention can then be subdivided further into:

1. planned delayed surgical placental delivery; or

2. planned conservative management of the placenta, i.e. leaving the placenta in-situ for resorption without surgical placental delivery (Perez-Delboy 2014).

The placenta sloughs off slowly over time, thus avoiding hysterectomy (ACOG 2012; Clausen 2013; Langhoff-Roos 2013; Merz 2009). Various case-reports and case-series have shown that conservative treatment is associated with lower maternal morbidity rates, 
but requires very close follow-ups (Lo 2014; Meyer 2012; Ramoni 2013; Torrega 2013; Tourette 2013). This approach avoids removal of the womb (hysterectomy), and could be useful in populations that are surgically conservative.

\section{One-step conservative surgery}

This method is described by Clausen et al. for focal accreta (Clausen 2014). It consists of cutting and removing the affected area in the womb together with the afterbirth and then reconstructing the womb muscles in a single 'one-step procedure' (Clausen 2014).

\section{The complete placental removal method}

The placenta is completely removed during surgery. This method can cause severe bleeding. It is not recommended at present ( Sentilhes 2013).

\section{Methotrexate}

Methotrexate is a drug that stops rapidly dividing cells from multiplying and growing (anti-folic acid properties). Evidence of the usage of this drug for managing MAP is controversial. The drug may decrease the amount of blood flow to the placenta. The placenta is slowly necrosed and expelled in small pieces (Wortman 2013). It is still unclear if this drug is useful on placental cells after delivery of the fetus, as these cells are not rapidly dividing. Women, allocated to this treatment, are perhaps at a risk of serious sideeffects and need close monitoring (Wortman 2013). Wortman et al. noted that, many women expelled or extruded their placenta, even without the using this drug (Wortman 2013). However, it may still be an option in a carefully selected group of women with MAP (Lin 2015).

\section{How these interventions might work}

Caesarean hysterectomy may significantly limit blood loss with controlled, planned delivery of the placenta. Leaving the placenta in-situ after planned caesarean delivery, allows for the body's natural resorption of the abnormal placental tissue, thus helping in uterine preservation. Similarly, one-step conservative surgery removes just the affected abnormal tissue. The complete placental removal method may also work by removing the affected placental tissue completely.

Methotrexate acts by stopping the abnormally invading placental tissue from dividing. The placenta is then resorbed over time, thus enabling uterine preservation.

\section{Description of other interventions and how these may work}

\section{Timing of delivery}

The proper time for delivery of pregnant women with MAP is controversial (Wright 2013). In a large American study of more than 500 practitioners, about $41 \%$ recommended delivery between 34 to 36 weeks (Wright 2013). The Royal College of Obstetricians and Gynaecologists (RCOG), suggest that uncomplicated cases can be delivered safely at around 36 to 37 weeks (RCOG 2011). Robinson et al. suggest delivery between 34 to 37 weeks (Robinson 2010).

Earlier delivery (e.g. 34 weeks) is perhaps safer for the mother and her child, as it might avoid massive haemorrhage or spontaneous labour, which is often seen with MAP (including placenta praevia) in the later weeks of pregnancy.

\section{Tertiary referral}

Referral to a tertiary level hospital and a multidisciplinary team involvement could help improve outcomes in women with MAP (Perez-Delboy 2014; Wortman 2013). Care in a tertiary facility might allow for better support in terms of maternal intensive care, blood transfusion, anaesthesia services and better care of the preterm neonate.

\section{Pre-operative planning protocol/checklist}

Preoperative checklists have been known to decrease maternal morbidity in these complicated surgeries (El-Messidi 2012; Table 1). Therefore, checklists and protocols may help organise the team by systematic preparation.

\section{Adjuvant measures}

The American College of Obstetricians and Gynecologists (ACOG) recommend that caesarean hysterectomy is safer when accompanied by other measures to arrest bleeding during surgery (ACOG 2012; Wortman 2013). Ureteral stents may help the clinician in identifying the ureter, to avoid ureteral injury during surgery.

Haemostatic brace suturing (as the B-lynch suture or modified compression sutures) may help resolve atony and cut the need for caesarean hysterectomy and blood transfusion by compressing the uterus.

Cell salvage may help by recycling the woman's blood and thereby reducing the need for blood transfusion. 


\section{Why it is important to do this review}

MAP is a condition that has serious adverse outcomes for the mum and her baby. There are a number of management options and mixed views on the ideal treatment, place of delivery and timing of delivery. Data from high-quality studies, need analysis in a thorough and systematic way, to check the usefulness and efficiency of different approaches for managing MAP.

\section{O B JECT IVES}

To assess the benefits and harms associated with clinical interventions for improving outcomes in women with antenatally diagnosed or suspected MAP. We will compare conventional caesarean hysterectomy with uterine conservation treatments (together with adjunct measures) in women who have been diagnosed antenatally with, or suspected to have, MAP.

\section{METHODS}

\section{Criteria for considering studies for this review}

\section{Types of studies}

Randomised controlled trials (RCTs) including those using quasirandomised and cluster-randomised designs will be eligible for inclusion. If just the abstract is available, we will contact the relevant author or authors for a copy of the paper and will include the study provisionally, as 'awaiting assessment'. Abstracts that have detailed statistics would be considered for inclusion. Cross-over studies would be excluded.

We plan to exclude studies where a diagnosis of MAP was not made or suspected in the antenatal period and all studies where the delivery method was not pre-planned.

\section{Types of participants}

The participants will be women who have had a confirmed or suspected diagnosis of MAP in the antenatal period (before 34 completed weeks of gestation). All definitions will be according to those used by the trial authors. If a study includes women with multiple pregnancies, their data would be included into the review and analysed according to the established criteria for multiple pregnancy.

\section{Types of interventions}

We plan to look into certain types of interventions and pair-wise comparisons as below, in our review (Higgins 2011).

\section{Interventions}

1. Planned caesarean hysterectomy.

2. Timing of delivery (e.g. $<36$ weeks versus $>=36$ weeks).

3. Tertiary care centre for delivery.

4. Pre-operative planning protocol or checklist or both.

5. Prophylactic occlusion balloon catheter (POBC) or uterine artery embolisation (UAE).

6. Ureteral stents.

7. Uterine compression sutures (B-Lynch, etc).

8. Cell salvage.

9. Conservative management; this includes leaving the placenta in-situ, one-step conservative surgery, or methotrexate.

\section{Comparisons}

1. Planned caesarean hysterectomy with or without adjuvant measures compared with conservative management (i.e. delivering the baby and preserving the uterus) with or without adjuvant measures.

2. Delivery before 36 weeks compared with delivery at or more than 36 weeks.

3. Delivery at a tertiary care centre versus delivery elsewhere.

4. Pre-operative planning protocol or checklist, or both, versus none.

5. Use of POBC or UAE versus none

6. Use of ureteral stents versus none.

7. Use of the uterine compression sutures (B-Lynch, etc) during caesarean section compared with none.

8. Using cell salvage therapy versus none.

We may however, have to change our comparisons or add new ones in the light of the type of data we collect (Higgins 2011).

\section{Types of outcome measures}

\section{Primary outcomes}

1. Maternal mortality.

2. Severe postpartum haemorrhage (blood loss of $1000 \mathrm{~mL}$ or more within 24 hours after birth).

3. Perinatal mortality (stillbirth and neonatal death).

\section{Secondary outcomes}

\section{Mother}

1. Caesarean hysterectomy rates.

2. Any other significant blood loss in pregnancy and the postpartum period (not included as a primary outcome above and according to trial authors definition/s).

3. Anaemia.

Interventions for improving pregnancy outcomes in antenatally diagnosed or suspected morbidly adherent placenta (Protocol) 
4. The number of units of blood transfused (and any other blood products).

5. Intensive care unit (ICU) admission or readmission.

6. Duration of hospitalisation.

7. Serious maternal morbidity (e.g. thromboembolism, coagulopathy, paralytic ileus, organ, injury: bladder or ureter or bowel or all, vesicovaginal fistula, sepsis, shock).

\section{Newborn}

1. Serious neonatal morbidity (e.g. respiratory distress syndrome, intraventricular haemorrhage, low Apgar scores, convulsions, neonatal anaemia, hypertonia, hypotonia; etc.).

2. Preterm birth (<37 weeks' gestation) rate.

3. Small-for-gestational age (SGA) (below 10th centile on customised birthweight chart, or as defined by trial authors).

4. Assisted ventilation of the newborn.

5. Low umbilical arterial blood $\mathrm{pH}$ (defined as less than 7.2, or as by the trial authors).

6. Neonatal intensive care unit (NICU) care.

Note: All definitions above will be according to those used by trial authors.

\section{Search methods for identification of studies}

The following methods section of this protocol is based on a standard template used by the Cochrane Pregnancy and Childbirth Group.

\section{Electronic searches}

We will search the Cochrane Pregnancy and Childbirth Group's Trials Register by contacting the Trials Search Co-ordinator.

The Register is a database containing over 20,000 reports of controlled trials in the field of pregnancy and childbirth. For full search methods used to populate the PCG Trials Register including the detailed search strategies for CENTRAL, MEDLINE, Embase and CINAHL; the list of handsearched journals and conference proceedings, and the list of journals reviewed via the current awareness service, please follow this link to the editorial information about the Cochrane Pregnancy and Childbirth Group in The Cochrane Library and select the 'Specialized Register' section from the options on the left side of the screen.

Briefly, the Cochrane Pregnancy and Childbirth Group's Trials Register is maintained by the Trials Search Co-ordinator and contains trials identified from:

1. monthly searches of the Cochrane Central Register of Controlled Trials (CENTRAL);

2. weekly searches of MEDLINE (Ovid);

3. weekly searches of Embase (Ovid);

4. monthly searches of CINAHL (EBSCO);
5. handsearches of 30 journals and the proceedings of major conferences;

6. weekly current awareness alerts for a further 44 journals plus monthly BioMed Central email alerts.

Search results are screened by two people and the full text of all relevant trial reports identified through the searching activities described above is reviewed. Based on the intervention described, each trial report is assigned a number that corresponds to a specific Pregnancy and Childbirth Group review topic (or topics), and is then added to the Register. The Trials Search Co-ordinator searches the Register for each review using this topic number rather than keywords. This results in a more specific search set that will be fully accounted for in the relevant review sections (Included, Excluded, Awaiting Classification or Ongoing).

In addition, we will search Open Grey, LILACS, HISA (Public Health); Popline, MedCarib (Caribbean Health Sciences literature), WPRIM (WHO Western Pacific region), Trip database and BASE.

We will also search ClinicalTrials.gov and the WHO International Clinical Trials Registry Platform (ICTRP) for unpublished, planned and ongoing trial reports.

See: Appendix 1 for the search terms, we plan to use for all these sources.

\section{Searching other resources}

We will search the reference lists of retrieved papers. We will contact colleagues and trial authors for any incomplete or unpublished data (if applicable).

We will not apply any language or date restrictions.

\section{Data collection and analysis}

We will use the methods as described in the Cochrane Handbook for Systematic Reviews of Interventions (Higgins 2011).

\section{Selection of studies}

At least two review authors from (RSG/AG/SQ/AB) will independently assess eligibility according to the inclusion criteria and evaluate the methodological quality of the potentially eligible studies (Higgins 2011). Any disagreement/s will be resolved by discussion between the review authors and where necessary, a third review author from (WF/VB/AA). We will contact authors of primary studies for clarification if necessary. If just the abstract is available, we will contact the relevant author/s for a copy of the paper and will include the study provisionally, as 'awaiting assessment'. Some studies where the abstract has detailed statistics and the paper is unobtainable may be included.

We will create a study flow diagram to map out the number of records identified, included and excluded directly into the Review Manager software (RevMan 2014). 


\section{Data extraction and management}

At least two review authors from (RSG/AG/SQ/AB) will extract the data and enter this into Review Manager 2014. We will resolve discrepancies through discussion or if required, we will consult (VB/WF/AA). We will enter data directly into the Review Manager Software (RevMan 2014) and check data for accuracy. When information regarding any of the above is unclear, we will attempt to contact authors of the original reports to provide further details (Higgins 2011).

\section{Assessment of risk of bias in included studies}

Review author pairs (from RSG/AG/SQ/AB) will independently assess risk of bias for each study using the criteria outlined in the Cochrane Handbook for Systematic Reviews of Interventions ( Higgins 2011). We will resolve any disagreement by discussion or by involving VB/WF/AA. Where necessary, study authors will be contacted for clarification.

\section{(I) Random sequence generation (checking for possible selection bias)}

We will describe for each included study the method used to generate the allocation sequence in sufficient detail to allow an assessment of whether it should produce comparable groups.

We will assess the method as:

- low risk of bias (any truly random process, e.g. random number table; computer random number generator);

- high risk of bias (any non-random process, e.g. odd or even date of birth; hospital or clinic record number);

- unclear risk of bias.

\section{(2) Allocation concealment (checking for possible selection} bias)

We will describe for each included study the method used to conceal allocation to interventions prior to assignment and will assess whether intervention allocation could have been foreseen in advance of, or during recruitment, or changed after assignment. We will assess the methods as:

- low risk of bias (e.g. telephone or central randomisation; consecutively numbered sealed opaque envelopes);

- high risk of bias (open random allocation; unsealed or nonopaque envelopes, alternation; date of birth);

- unclear risk of bias.

\section{(3.I) Blinding of participants and personnel (checking for possible performance bias)}

We will describe for each included study the methods used, if any, to blind study participants and personnel from knowledge of which intervention a participant received. We will consider that studies are at low risk of bias if they were blinded, or if we judge that the lack of blinding would be unlikely to affect results. We will assess blinding separately for different outcomes or classes of outcomes.

We will assess the methods as:

- low, high or unclear risk of bias for participants;

- low, high or unclear risk of bias for personnel.

\section{(3.2) Blinding of outcome assessment (checking for possible detection bias)}

We will describe for each included study the methods used, if any, to blind outcome assessors from knowledge of which intervention a participant received. We will assess blinding separately for different outcomes or classes of outcomes.

We will assess methods used to blind outcome assessment as:

- low, high or unclear risk of bias.

(4) Incomplete outcome data (checking for possible attrition bias due to the amount, nature and handling of incomplete outcome data)

We will describe for each included study, and for each outcome or class of outcomes, the completeness of data including attrition and exclusions from the analysis. We will state whether attrition and exclusions were reported and the numbers included in the analysis at each stage (compared with the total randomised participants), reasons for attrition or exclusion where reported, and whether missing data were balanced across groups or were related to outcomes. Where sufficient information is reported, or can be supplied by the trial authors, we will re-include missing data in the analyses which we undertake (Higgins 2011).

We will assess methods as:

- low risk of bias (e.g. no missing outcome data; missing outcome data balanced across groups);

- high risk of bias (e.g. numbers or reasons for missing data imbalanced across groups; 'as treated' analysis done with substantial departure of intervention received from that assigned at randomisation);

- unclear risk of bias.

We would prefer to use a cut-off point of $20 \%$, which is the most commonly used value (Higgins 2011 See Handbook section 8.13).

\section{(5) Selective reporting (checking for reporting bias)}

We will describe for each included study how we investigated the possibility of selective outcome reporting bias and what we found. We will assess the methods as:

- low risk of bias (where it is clear that all of the study's prespecified outcomes and all expected outcomes of interest to the review have been reported); 
- high risk of bias (where not all the study's pre-specified outcomes have been reported; one or more reported primary outcomes were not pre-specified; outcomes of interest are reported incompletely and so cannot be used; study fails to include results of a key outcome that would have been expected to have been reported);

- unclear risk of bias.

\section{(6) Other bias (checking for bias due to problems not} covered by (I) to (5) above)

We will describe for each included study any important concerns we have about other possible sources of bias. These will be:

- if a potential source of bias was related to the specific study design?

- if there was extreme baseline imbalance?

- if the study has been claimed to be fraudulent?

- if the trial was stopped early due to some data-dependent process?

(Higgins 2011 See Handbook section 8.15).

We will assess whether each study was free of other problems that could put it at risk of bias:

- low risk of other bias;

- high risk of other bias;

- unclear whether there is risk of other bias.

\section{(7) Overall risk of bias}

We will make explicit judgements about whether studies are at high risk of bias, according to the criteria given in the Handbook (Higgins 2011). With reference to (1) to (6) above, we will assess the likely magnitude and direction of the bias and whether we consider it is likely to impact on the findings.

\section{(8) Cluster-randomised studies}

We will follow the guidance in section 16.3.2 Cochrane Handbook for Systematic Reviews of Interventions (Higgins 2011) to assess particular biases in relation to cluster-randomised trials.

\section{Assessing the quality of the body of evidence using the GRADE approach}

We plan to include a 'Summary of findings' table for the main (primary) outcomes listed above. We shall use the GRADEpro (GRADEproGDT) (GRADEpro 2014) to import data from Review Manager 5.3 (RevMan 2014) in order to create a 'Summary of findings' table (Higgins 2011).

\section{Assessing the quality of the evidence using the GRADE approach}

The quality of the evidence will be assessed using the GRADE approach as outlined in the GRADE handbook in order to assess the quality of the body of evidence relating to the following outcomes for the main comparisons.

1. Maternal mortality.

2. Severe postpartum haemorrhage (blood loss of $1000 \mathrm{~mL}$ or more within 24 hours after birth).

3. Perinatal mortality (stillbirth and neonatal death).

4. Caesarean hysterectomy rates

5. Intensive care unit (ICU) admission or readmission (for the mother)

6. Duration of hospitalisation (for the mother) The main comparisons for use in GRADE are listed below.

1. Planned caesarean hysterectomy with or without adjuvant measures compared with conservative management (i.e. delivering the baby and preserving the uterus) with or without adjuvant measures.

2. Delivery before 36 weeks compared with delivery at or more than 36 weeks.

3. Delivery at a tertiary care centre versus delivery elsewhere.

4. Pre-operative planning protocol or checklist, or both, versus none.

5. Use of POBC or UAE versus none.

6. Use of ureteral stents versus none.

7. Use of the uterine compression sutures (B-Lynch, etc) during caesarean section compared with none.

8. Using cell salvage therapy versus none. GRADEpro Guideline Development Tool will be used to import data from Review Manager 5.3 (RevMan 2014) in order to create 'Summary of findings' tables. A summary of the intervention effect and a measure of quality for each of the above outcomes will be produced using the GRADE approach. The GRADE approach uses five considerations (study limitations, consistency of effect, imprecision, indirectness and publication bias) to assess the quality of the body of evidence for each outcome. The evidence can be downgraded from 'high quality' by one level for serious (or by two levels for very serious) limitations, depending on assessments for risk of bias, indirectness of evidence, serious inconsistency, imprecision of effect estimates or potential publication bias.

\section{Measures of treatment effect}

\section{Dichotomous data}

For dichotomous data, we will present results as a summary risk ratio (RR) with 95\% confidence intervals (Higgins 2011). 


\section{Continuous data}

For continuous data, we will use the mean difference (MD) if outcomes are measured in the same way between trials. We will use the standardised mean difference (SMD) to combine trials that measure the same outcome, but use different methods (Higgins 2011).

\section{Unit of analysis issues}

\section{Cluster-randomised trials}

We will include cluster-randomised trials in the analyses along with individually-randomised trials. We will adjust the sample sizes using the methods described in the Handbook (Higgins 2011) using an estimate of the intra cluster correlation co-efficient (ICC) derived from the trial, or from a similar trial or from a study of a similar population.

If we identify both cluster-randomised trials and individually-randomised trials, we plan to synthesise the relevant information. We will consider it reasonable to combine the results from both if there is little heterogeneity between the study designs and the interaction between the effect of intervention and the choice of randomisation unit is considered to be unlikely (Higgins 2011). We will also acknowledge heterogeneity in the randomisation unit and perform a subgroup analysis to investigate the effects of the randomisation unit (Higgins 2011).

\section{Cross-over trials}

Cross-over trials will not be included in this review.

\section{Other unit of analysis issues}

For studies that included multiple arms, three arms for example, we will include these if any pair-wise comparisons if the intervention groups are relevant to the review. However, where one arm appears more than once on the same meta-analysis, the outcome and denominator will be divided by the number of times it appears, to avoid multiple counting. For example, if the number of events in the control group is an odd number, to reduce the risk of overestimating effects in favour of the intervention group, we will then halve it and round it down. For odd denominators (total number of participants in the control group), we will round these numbers upwards for the same reason (Higgins 2011).

\section{Dealing with missing data}

For included studies, we will note levels of attrition. Whenever possible, the lead review author will contact the trial authors to request missing data. Potential impact of missing data (known or suspected) on findings of the review will be addressed in the 'Discussion' section.
For all outcomes, we will carry out analyses, as far as possible, on an intention-to-treat basis, i.e. we will attempt to include all participants randomised to each group in the analyses and all participants will be analysed in the group to which they were allocated, regardless of whether or not they received the allocated intervention. The denominator for each outcome in each trial will be the number randomised minus any participants whose outcomes are known to be missing.

We will carry out a sensitivity analysis (see below) instead of completely excluding trials with missing data.

\section{Assessment of heterogeneity}

We will assess statistical heterogeneity in each meta-analysis using the $\mathrm{Tau}^{2}, \mathrm{I}^{2}$ and $\mathrm{Chi}^{2}$ statistics. We will regard heterogeneity as substantial if an $\mathrm{I}^{2}$ is greater than $30 \%$ and either the $\mathrm{Tau}^{2}$ is greater than zero, or there is a low P value (less than 0.10 ) in the $\mathrm{Chi}^{2}$ test for heterogeneity (Higgins 2011).

\section{Assessment of reporting biases}

If there are 10 or more studies in the meta-analysis, we will investigate reporting biases (such as publication bias) using funnel plots. We will assess funnel plot asymmetry visually. If asymmetry is suggested by a visual assessment, we will perform exploratory analyses to investigate it and to investigate whether asymmetry is the result of small-study effects, publication bias, or other factors. If it is likely that asymmetry is caused by small-study effects, we will conduct sensitivity analysis to explore how this affects the results and conclusions of the meta-analysis.

\section{Data synthesis}

We will carry out statistical analysis using the Review Manager software (RevMan 2014). We will use fixed-effect model for combining data where it is reasonable to assume that studies are estimating the same underlying treatment effect; i.e. where trials are examining the same intervention, and the trials' populations and methods are judged sufficiently similar. If clinical heterogeneity is sufficient to expect that the underlying treatment effects differ between trials, or if substantial statistical heterogeneity is detected, we will use the random-effects model to produce an overall summary (RevMan 2014).

The random-effects summary will be treated as the average of the range of possible treatment effects and we will discuss the clinical implications of treatment effects differing between trials. If the average treatment effect is not clinically meaningful, we will not combine trials.

If we use the random-effects analyses, the results will be presented as the average treatment effect with $95 \%$ confidence intervals and the estimates of $\mathrm{Tau}^{2}$ and $\mathrm{I}^{2}$. 


\section{Subgroup analysis and investigation of heterogeneity}

If we find significant heterogeneity, we will conduct a subgroup analyses. We will consider whether an overall summary is useful, and if it is, use random-effects analysis to produce it.

We plan to carry out the following subgroup analyses.

1. Previous caesarean births (none versus one or more).

2. Multiple pregnancy versus singleton pregnancy.

3. Total versus subtotal hysterectomy.

4. Elective versus emergency caesarean.

We will plan a subgroup analysis for all outcomes within the main analysis.

We will assess subgroup differences by interaction tests available within RevMan (RevMan 2014). We will report the results of subgroup analyses quoting the $\mathrm{Chi}^{2}$ statistic and $\mathrm{P}$ value, and the interaction test $\mathrm{I}^{2}$ value (Higgins 2011). We will contact trial authors directly if we have any questions regarding their data (Higgins 2011).

\section{Sensitivity analysis}

We plan to carry out sensitivity analyses to explore the effect of trial quality and small-study effects by excluding studies with risk of bias and small sample size concerns from the analyses. This is to decide, whether this will make any difference, to the overall result. 'High quality' trials chosen will be those with an adequate sequence generation, where the allocation was adequately concealed and those with an attrition rate (drop out) of less than of 20\% (low risk bias trials) (Higgins 2011).

In summary, we plan to carry out a sensitivity analysis by examining the effect on the findings, by excluding the following.
1. Trials at high risk of bias based on allocation concealment or quasi-randomised trials.

2. Trials with small sample sizes.

3. Trials with a high attrition rate (>20\%).

The high risk domains that we plan to look into will include (Higgins 2011) the following:

1. age range of the participants;

2. dosage of drugs used as methotrexate;

3. criteria used to define the comparator group;

4. time lines for the outcomes.

We will not exclude any missing data, but consider a sensitivity analysis instead. We will contact trial authors directly if we have any questions regarding their data (Higgins 2011).

\section{ACKNOWLEDGEMEN TS}

We would like to thank the Cochrane Pregnancy and childbirth Group for their help with the review protocol.

As part of the pre-publication editorial process, this protocol has been commented on by three peers (an editor and two referees who are external to the editorial team) and the Group's Statistical Adviser.

This project was supported by the National Institute for Health Research, via Cochrane Infrastructure funding to Cochrane Pregnancy and Childbirth. The views and opinions expressed therein are those of the authors and do not necessarily reflect those of the Systematic Reviews Programme, NIHR, NHS or the Department of Health.

\section{RE F E R E N C E S}

\section{Additional references}

\section{ACOG 2012}

American College of Obstetricians and Gynecologists. Placenta accreta committee opinion no. 529. Obstetrics and Gynecology 2012;120(1):207-11.

\section{Al-Khan 2014}

Al-Khan A, Gupta V, Illsley NP, Mannion C, Koenig C, Bogomol A, et al. Maternal and fetal outcomes in placenta accreta after institution of team-managed care. Reproductive Sciences 2014;21(6):761-71.

Amsalem 2011

Amsalem H, Kingdom JC, Farine D, Allen L, Yinon Y, D'Souza DL, et al. Planned caesarean hysterectomy versus "conserving" caesarean section in patients with placenta accreta. Journal of Obstetrics and Gynaecology Canada 2011; 33(10):1005-10.

\section{Berkley 2013}

Berkley EM, Abuhamad AZ. Prenatal diagnosis of placenta accreta: is sonography all we need?. Journal of Ultrasound In Medicine 2013;32(8):1345-50.

\section{Bowman 2014}

Bowman ZS, Eller AG, Bardsley TR, Greene T, Varner MW, Silver RM. Risk factors for placenta accreta: a large prospective cohort. American Journal of Perinatology 2014; 31(9):799-804.

\section{Cavallaro 2013}

Cavallaro FL, Cresswell JA, Franca GVA, Victora CG, Barros AJD, Ronsmans C. Trends in caesarean delivery by country and wealth quintile: cross-sectional surveys in southern Asia and sub-Saharan Africa. Bulletin of The World Health Organization 2013;91(12):914-22D.

\section{Clausen 2013}

Clausen C, Stensballe J, Albrechtsen CK, Hansen MA, Lonn L, Langhoff-Roos J. Balloon occlusion of the internal iliac 
arteries in the multidisciplinary management of placenta percreta. Acta Obstetricia et Gynecologica Scandinavica 2013; 92(4):386-91.

\section{Clausen 2014}

Clausen C, Lonn L, Langhoff-Roos J. Management of placenta percreta: a review of published cases. Acta Obstetricia et Gynecologica Scandinavica 2014;93(2): 138-43.

\section{Comstock 2014}

Comstock $\mathrm{CH}$, Bronsteen RA. The antenatal diagnosis of placenta accreta. British Journal of Obstetrics and Gynaecology 2014;121:171-82.

\section{El-Messidi 2012}

El-Messidi A, Mallozzi A, Oppenheimer L. A multidisciplinary checklist for management of suspected placenta accreta. Journal Of Obstetrics and Gynaecology Canada 2012;34(4):320-4.

\section{Eller 2011}

Eller AG, Bennett MA, Sharshiner M, Masheter C, Soisson AP, Dodson M, et al. Maternal morbidity in cases of placenta accreta managed by a multidisciplinary care team compared with standard obstetric care. Obstetrics and Gynaecology. 2011;117(2 Pt 1):331-7. [PUBMED: 21309195]

\section{Fitzpatrick 2014}

Fitzpatrick KE, Sellers S, Spark P, Kurinczuk JJ, Brocklehurst P, Knight M. The management and outcomes of placenta accreta, increta, and percreta in the UK a populationbased descriptive study. British Journal of Obstetrics and Gynaecology 2014;121(1):62-70. [DOI: 10.1111/ 1471-0528.12405]

\section{Garmi 2012}

Garmi G, Salim R. Epidemiology, etiology, diagnosis, and management of placenta accreta. Obstetrics and Gynecology International 2012;2012:1-44.

\section{Hayes 2011}

Hayes E, Ayida G, Crocker A. The morbidly adherent placenta: diagnosis and management options. Current Opinion in Obstetrics and Gynecology 2011;23(6):448-53.

\section{Herath 2012}

Herath RP, Wijesinghe PS. Management of morbidly adherent placenta. Sri Lankan Journal of Obstetrics and Gynaecology 2012;33(2):39-44.

\section{Higgins 2011}

Higgins JPT, Green S, editors. Cochrane Handbook for Systematic Reviews of Interventions Version 5.1.0 (updated March 2011). The Cochrane Collaboration, 2011. Available from handbook.cochrane.org.

\section{Jauniaux 2012}

Jauniaux E, Jurkovic D. Placenta accreta pathogenesis of a 20th century iatrogenic uterine disease. Placenta 2012;33 (4):244-51.

\section{Langhoff-Roos 2013}

Langhoff-Roos J, Chantraine F, Omas Geirsson RT. AIP (abnormally invasive placenta) - from a retained placenta to destruction of the uterine wall (Editors message). Acta Obstetricia et Gynecologica Scandinavica 2013;92(4):367-8.

Lin 2015

Lin K, Qin J, Xu K, Hu W, Lin J. Methotrexate management for placenta accreta: a prospective study. Archives of Gynecology and Obstetrics 2015;291(6):1259-64.

Lo 2014

Lo TK, Yung WK, Lau WL, Law B, Lau S, Leung WC.

Planned conservative management of placenta accreta - experience of a regional general hospital. Journal of Maternal-Fetal and Neonatal Medicine 2014;27(3):291-6.

\section{Merz 2009}

Merz W, Van de Vondel P, Strunk H, Geipel A, Gembruch U. Diagnosis, treatment and application of colour doppler in conservative management of abnormally adherent placenta. Ultraschall In der Medizin 2009;30(6):571-6.

\section{Meyer 2012}

Meyer NP, Ward GH, Chandraharan E. Conservative approach to the management of morbidly adherent placentae. Ceylon Medical Journal 2012;57(1):36-9.

\section{Palacios-Jaraquemada 2013}

Palacios-Jaraquemada JM, Bruno CH, Martin E. MRI in the diagnosis and surgical management of abnormal placentation. Acta Obstetricia et Gynecologica Scandinavica 2013;92(4):392-7.

\section{Perez-Delboy 2014}

Perez-Delboy, Wright J. Surgical management of placenta accreta: to leave or remove the placenta?. British Journal of Obstetrics and Gynaecology 2014;121(2):163-70.

\section{Ramoni 2013}

Ramoni A, Strobl E, Tiechl J, Ritter M, Marth C. Conservative management of abnormally invasive placenta:four case reports. Acta Obstetricia et Gynecologica Scandinavica 2013;92(4):468-71. [DOI: 10.1111/ aogs. 12079]

\section{RCOG 2011}

Johnston TA, Paterson-Brown S. Placenta praevia, placenta praevia accreta and vasa previa: diagnosis and management. Royal College of Obstetricians and Gynaecologists (RCOG) Green-Top Guideline No.27. London, UK: RCOG, 2011 (updated 2014):1-26.

\section{RevMan 2014 [Computer program]}

The Nordic Cochrane Centre, The Cochrane Collaboration. Review Manager (RevMan). Version 5.3. Copenhagen: The Nordic Cochrane Centre, The Cochrane Collaboration, 2014.

\section{Robinson 2010}

Robinson B, Grobman W. Effectiveness of timing strategies for delivery of individuals with placenta previa and accreta. Obstetrics and Gynecology 2010;116(4):835-42.

\section{Sentilhes 2013}

Sentilhes L, Goffinet F, Kayem G. Management of placenta accreta. Acta Obstetricia et Gynecologica Scandinavica 2013; 92(10):1125-34. 
Tan 2013

Tan G, Jobling TW, Wallace EM, McNeilage LJ, Manolitsas

T, Hodges RJ. Surgical management of placenta accreta:

a 10-year experience. Acta Obstetricia et Gynecologica

Scandinavica 2013;92(4):445-50.

Torrega 2013

Torrenga B, Huirne JA, Bolte AC, Van Waesberghe JHTM, De Vries JIP. Postpartum monitoring of retained placenta: two cases of abnormally adherent placenta. Acta Obstetricia et Gynecologica Scandinavica 2013;92(4):472-5.

Tourette 2013

Tourette C, Bretelle F, Cravello L, D’Ercole C, Boubli L, Gamerre M, et al. Comparative study of patients with placenta accreta with or without a history of cesarean section. Journal de Gynecologie Obstetrique et Biologie de la Reproduction 2013;43(4):322-7.

Upson 2014

Upson K, Silver RM, Greene R, Lutomski J, Holt VL. Placenta accreta and maternal morbidity in the Republic of Ireland, 2005-2010. Journal of Maternal Fetal and Neonatal Medicine 2014;27(1):24-9.

\section{Vogel 2015}

Vogel P, Betrán AP, Vindevoghel N, Souza JP, Torloni MR, Zhang J, et al. Use of the Robson classification to assess caesarean section trends in 21 countries: a secondary analysis of two WHO multicountry surveys. Lancet Global Health 2015;3(5):e260-e270.

\section{Weiniger 2013}

Weiniger CF, Einav S, Deutsch L, Ginosar Y, Ezra Y, Eid L. Outcomes of prospectively-collected consecutive cases of antenatal-suspected placenta accreta. International Journal of Obstetric Anesthesia 2013;22(4):273-9.

\section{WHO 2015}

World Health Organization (WHO). WHO statement on caesarean section rates. http://apps.who.int/iris/bitstream/ 10665/161442/1/WHO_RHR_15.02_eng.pdf?ua =1 (accessed 5 February 2016) 2015.

\section{Wortman 2013}

Wortman AC, Alexander JM. Placenta accreta, increta, and percreta. Obstetrics and Gynecology Clinics of North America 2013;40(1):137-54.

\section{Wright 2013}

Wright JD, Silver RM, Bonanno C, Gaddipati S, Lu YS, Simpson LL, et al. Practice patterns and knowledge of obstetricians and gynecologists regarding placenta accreta. Journal of Maternal-Fetal and Neonatal Medicine 2013;26 (16):1602-9.

* Indicates the major publication for the study

\title{
ADDITIONAL TABLES
}

Table 1. A multidisciplinary checklist for management of suspected placenta accreta (from Checklist from El-Messidi 2010)

\author{
Not applicable To do Pending Complete
}

Present/Absent

\section{ULTRASOUND}

Details of placentation:

\begin{tabular}{l} 
Anterior \\
Posterior \\
\hline LLP or previa \\
\hline $\begin{array}{l}\text { Loss of echo lucency be- } \\
\text { tween uterus and pla- } \\
\text { centa }\end{array}$ \\
Lacunae \\
\hline $\begin{array}{l}\text { Interruption of bladder- } \\
\text { uterine interface }\end{array}$
\end{tabular}

Interventions for improving pregnancy outcomes in antenatally diagnosed or suspected morbidly adherent placenta (Protocol) 
Table 1. A multidisciplinary checklist for management of suspected placenta accreta (from Checklist from El-Messidi 2010) (Continued)

Suspected accreta by colour Doppler

DESIGNATED

DELIVERY CENTRE

MRI Consultations.

MFM team

Anaesthesia

Interventional radiology

Most experienced sur-

geons (e.g., gyn-oncol-

ogy)

Urology

Neonatal ICU

LABORATORY Most recent date:

2 to 4 units PRBCs currently on hold

CBC

Coagulation profile

INTRAOPERATIVE

PLANS

Notification of the main

OR (operating theatre)

\section{Consent form}

Preoperative internal il-

iac stents

4 units PRBC(packed cells) on hold (or as requested)

Hysterectomy tray available 
Table 1. A multidisciplinary checklist for management of suspected placenta accreta (from Checklist from El-Messidi 2010) (Continued)

Cystoscopy set available/ urology team

Cell Saver

Neonatal team present

Experienced surgeons on

site

\section{A P PENDICES}

\section{Appendix I. Search terms}

Open Grey, LILACS, HISA (Public Health); Popline, MedCarib (Caribbean Health Sciences literature), WPRIM (WHO Western Pacific region), Trip database and BASE.

("adherent placenta” OR accreta OR increta OR percreta OR "placental attachment disorder").

ClinicalTrials.gov and the WHO International Clinical Trials Registry Platform (ICTRP):

We plan to search using simple terms for these databases. Each term will be searched separately and manually deduplicated: adherent placenta

accreta

increta

percreta

placental attachment disorder

\section{CONTRIBUTIONSOFAUTHORS}

Rinku Sengupta had the initial idea for undertaking the review and is guarantor for this review. Rinku Sengupta wrote the initial draft of this protocol.

All authors (Rinku Sengupta, Alexis Gimovsky, Shuqin Wei, Anthony Bamigboye, Vincenzo Berghella, Amanda Amorim Adegboye and William Fraser) reviewed and consented to or recommended changes to further drafts. 


\section{DECLARATIONSOF INTEREST}

Rinku Sengupta: none known

Alexis Gimovsky: none known

Shuqin Wei: none known

Anthony A Bamigboye: none known

Vincenzo Berghella: none known

Amanda R Amorim Adegboye: none known

William D Fraser: none known 